Article

\title{
The Topological Pressure of Linear Cellular Automata
}

\author{
Jung-Chao Ban ${ }^{1,2}$ and Chih-Hung Chang ${ }^{3, \star}$ \\ ${ }^{1}$ Department of Applied Mathematics, National Dong Hwa University, 97063, Hualian, Taiwan, \\ R.O.C.; E-mails: jcban@mail.ndhu.edu.tw \\ ${ }^{2}$ Taida Institute for Mathematical Sciences, National Taiwan University, 10617, Taipei, Taiwan, R.O.C. \\ ${ }^{3}$ National Center for Theoretical Sciences, Mathematics Division, National Tsing Hua University, \\ 30043, Hsinchu, Taiwan, R.O.C.
}

* Author to whom correspondence should be addressed; E-mail: chihhung@ math.cts.nthu.edu.tw

Received: 13 April 2009/Accepted: 6 May 2009 / Published: 11 May 2009

\begin{abstract}
This elucidation studies ergodicity and equilibrium measures for additive cellular automata with prime states. Additive cellular automata are ergodic with respect to Bernoulli measure unless it is either an identity map or constant. The formulae of measure-theoretic and topological entropies can be expressed in closed forms and the topological pressure is demonstrated explicitly for potential functions that depend on finitely many coordinates. According to these results, Parry measure is inferred to be an equilibrium measure.
\end{abstract}

Keywords: Cellular automata; additive; equilibrium measure.

MSC: 28D20, 37B15, 37B40, 47A35

\section{Introduction}

Cellular automaton (CA) is a particular class of dynamical systems introduced by Ulam [1] and von Neumann [2] as a model for self-production and is widely studied in a variety of contexts in physics, biology and computer science [3-11].

One-dimensional CA consists of infinite lattice with finite states and an associated mapping, say local rule. Hedlund [12] discusses CA systematically from purely mathematical point of view. Wolfram $[13,14]$ also makes a decisive impulse to the mathematical study; he proposes a classification of CA by means of asymptotical dynamics. Also, Wolfram does lots of computer simulation on those CA with 2 
states whose local rule is only related to its nearest neighbors in his book [15]. He numbers these 256 rules and divides them into four classes according to their asymptotic behavior. Chua et al. [16] assert that there are only 88 equivalent classes. Any two local rules in same equivalent class are topological conjugate to each other. In other words, local rules in same class admit the same dynamics and preserve invariants such as topological entropy, ergodicity, mixing, and so on.

Among these 256 rules, there are eight of them being additive. More precisely, those local rules can be represented as linear combination. They are indexed as 0,60,90, 102, 150, 170, 204, 240 respectively. When periodic boundary condition is considered, Chua et al. [17] investigate the dynamical behavior of these rules such as Isles of Eden, period of attractors, and so on [17, 18]. This demonstrates that additive CA can still propose rich dynamics.

This essay elucidates ergodicity and equilibrium measures for additive CA associated with prime states. Shirvani and Rogers [19] demonstrate that a one-dimensional two states CA is ergodic provided its local rule is either rightmost or leftmost permutive. This fact has been rediscovered by Shereshevsky [20, 21] and Kleveland [22] for permutive CA. Cattaneo et al. [23] extend their result to multi-dimensional additive CA with arbitrary finite alphabet. Additive CA whose states consist of prime symbols is a subclass of permutive CA, Theorem 7 asserts a different proof for the ergodicity via a construction method.

Ward [24] studies the topological entropy for additive CA with prime states. Akin [25] shows that the uniform Bernoulli measure is a measure with maximal entropy if the local rule $f$ is given by $f\left(x_{-k}, \ldots, x_{k}\right)=\sum_{i=-k}^{k} x_{i}$ for some $k \in \mathbb{N}$. Whenever an additive CA is an automorphism, Berg demonstrates that the uniform Bernoulli measure is the unique measure which maximizes the measuretheoretic entropy [26]. This investigation gives an alternative proof for the topological entropy and the measure-theoretic entropy of additive CA is demonstrated for any Bernoulli measure. Corollaries 1 and 2 give closed formulae for measure-theoretic and topological entropies respectively, here a Bernoulli measure is considered. Theorems 8 and 9 investigate the topological pressure for those potential functions that depend on finitely many coordinates. In addition, Parry measure is indicated to be an equilibrium measure. This generalizes Akin's result.

The rest of this paper is organized as follows. Section 2. states some notations and definitions. Section 3. studies the measure-theoretic and topological entropies while Section 4. investigates the ergodicity and the topological pressure. Section 5. extends the results to additive CA with prime symbols.

\section{Notation and Definition}

Let $\mathcal{A}=\{0,1\}$ be a finite alphabet and let $\Omega=\mathcal{A}^{\mathbb{Z}}$ be the space of infinite sequence $x=\left(x_{n}\right)_{-\infty}^{\infty}$. Hedlund [12] studies $\mathrm{CA}$ in the viewpoint of symbolic dynamics.

Theorem 1 ([12]). A map $F: \Omega \rightarrow \Omega$ is a $C A$ if and only if $F$ can be represented as a sliding block code, i.e., there exists $k \in \mathbb{Z}^{+}$and a block map $f: \mathcal{A}^{2 k+1} \rightarrow \mathcal{A}$ such that

$$
F(x)_{i}=f\left(x_{i-k}, \ldots, x_{i+k}\right)
$$

for $x \in \Omega$ and $i \in \mathbb{Z}$. 
Such $f$ is called the local rule of $F$. The study of the local rule of a CA is essential for the understanding of this system. A local rule $f: \mathcal{A}^{2 k+1} \rightarrow \mathcal{A}$ is called leftmost (respectively rightmost) permutive if there exists an integer $i,-k \leq i \leq-1$ (respectively $1 \leq i \leq k$ ), such that

(i) $f$ is a permutation at $x_{i}$ whenever the other variables are fixed;

(ii) $f$ does not depend on $x_{j}$ for $j<i$ (respectively $j>i$ ).

In the rest of this investigation, a particular class of CA in [15], say additive CA, is investigated and the results can be extended to any alphabet of prime symbols. The local rule of additive CA is defined by $f\left(x_{-1}, x_{0}, x_{1}\right)=\lambda_{-1} x_{-1}+\lambda_{0} x_{0}+\lambda_{1} x_{1} \bmod 2$, where $\lambda_{i}=0,1$ for $-1 \leq i \leq 1$. The correspondence between the local rules and Wolfram's rules are listed in the following.

\begin{tabular}{c|c|c} 
Local rules $f$ & Wolfram's rules & Equivalent rules \\
\hline 0 & 0 & 255 \\
$x_{1}$ & 170 & 240 \\
$x_{0}$ & 204 & \\
$x_{-1}$ & 240 & 170 \\
$x_{0}+x_{1}$ & 102 & $60,153,195$ \\
$x_{-1}+x_{1}$ & 90 & 165 \\
$x_{-1}+x_{0}$ & 60 & $102,153,195$ \\
$x_{-1}+x_{0}+x_{1}$ & 150 &
\end{tabular}

Rules 0,204 and 255 are called "trivial rules" because of their simple dynamics.

For any $m \geq 1, f$ can be extended to the mapping $f_{m}: \mathcal{A}^{m+2} \rightarrow \mathcal{A}^{m}$ by

$$
f_{m}\left(x_{-1}, \ldots, x_{m}\right)=\left(f\left(x_{-1}, x_{0}, x_{1}\right), \ldots, f\left(x_{m-2}, x_{m-1}, x_{m}\right)\right),
$$

here $f_{1}=f$.

Let $\mathcal{B}$ be the Borel $\sigma$-algebra on $\Omega$ and let $\mu=\left(p_{0}, p_{1}\right)$ be a Bernoulli measure. For any finite measurable partition $\alpha$ of $\Omega$, denote by

$$
H_{\mu}(\alpha)=-\sum_{A \in \alpha} \mu(A) \log \mu(A)
$$

The measure-theoretic entropy of $F$ with respect to $\alpha$ is defined by

$$
h_{\mu}(F, \alpha)=\lim _{n \rightarrow \infty} \frac{1}{n} H_{\mu}\left(\bigvee_{i=0}^{n-1} F^{-i} \alpha\right)
$$

where

$$
\mathcal{P} \bigvee \mathcal{Q}=\{P \cap Q: P \in \mathcal{P}, Q \in \mathcal{Q}\}
$$

Reader may refer to [27] for more details. The measure-theoretic entropy of $F$ is defined by

$$
h_{\mu}(F)=\sup h_{\mu}(F, \alpha)
$$

where the supremum is taken over all finite measurable partitions of $\Omega$. 
Define $d: \Omega \times \Omega \rightarrow \mathbb{R}$ by

$$
d(x, y)=\sum_{i=-\infty}^{\infty} \frac{\left|x_{i}-y_{i}\right|}{2^{|i|}}, \quad x, y \in \Omega
$$

It is easy to verify that $d$ is a metric and $(\Omega, d)$ is a compact metric space.

Let $\mathcal{P}$ be an open cover of $\Omega$, denote by

$$
H(\mathcal{P})=\inf \{\log |\hat{\mathcal{P}}|\}
$$

where the infimum is taken over the set of finite subcovers $\hat{\mathcal{P}}$ of $\mathcal{P}$ and $|A|$ is the cardinality of $A$. The topological entropy of $F$ with respect to $\mathcal{P}$ is defined by

$$
h(F, \mathcal{P})=\lim _{n \rightarrow \infty} \frac{1}{n} H\left(\bigvee_{i=0}^{n-1} F^{-i} \mathcal{P}\right) .
$$

The topological entropy of $F$ is defined by

$$
h_{\text {top }}(F)=\sup h(F, \mathcal{P})
$$

where the supremum is taken over all open covers of $\Omega$.

In addition, for $\alpha$ an open cover of $\Omega$ and $\phi \in C(\Omega, \mathbb{R})$ a continuous function, denote by

$$
p_{n}(F, \phi, \alpha)=\inf \left\{\sum_{B \in \beta} \sup _{x \in B} e^{\left(S_{n} \phi\right)(x)}: \beta \text { is a finite subcover of } \bigvee_{i=0}^{n-1} F^{-i} \alpha\right\} \text {, }
$$

where $n \in \mathbb{N}$ and $S_{n} \phi=\sum_{i=0}^{n-1} \phi \circ F^{i}$. Then $\lim _{n \rightarrow \infty} \frac{1}{n} \log p_{n}(F, \phi, \alpha)$ exists [27]. For each $\delta>0$, define

$$
P(F, \phi, \delta)=\sup \left\{\lim _{n \rightarrow \infty} \frac{1}{n} \log p_{n}(F, \phi, \alpha): \operatorname{diam}(\alpha) \leq \delta\right\},
$$

and

$$
P(F, \phi)=\lim _{\delta \rightarrow 0} P(F, \phi, \delta) .
$$

The map $P(F, \cdot): C(\Omega, \mathbb{R}) \rightarrow \mathbb{R} \cup\{\infty\}$ is called the topological pressure of $F$. It comes immediately that $P(F, 0)=h_{\text {top }}(F)$.

\section{Measures with Maximal Entropy}

Let $X$ be a compact metric space and let $T: X \rightarrow X$ be a continuous function. The variational principle says that the supremum of measure-theoretic entropy of $T$ coincides with the topological entropy of $T$, where the supremum is taken over all ergodic measures. That is to say, $h_{\text {top }}(T)=\sup \left\{h_{\mu}(T)\right.$ : $\mu$ is ergodic $\}$. A measure that reaches the supremum is called a measure with maximal entropy.

The goal of this section is going for the determination of measures with maximal entropy via the study of measure-theoretic and topological entropies. 


\subsection{Measure-theoretic entropy}

In this section, a closed formula of the measure-theoretic entropy is given.

Given two integers $r \leq s$ and $a_{r}, \ldots, a_{s} \in\{0,1\}$. Denote by $C(r, s)={ }_{r}\left[a_{r}, \ldots, a_{s}\right]_{s}$ a cylinder of $\Omega$, i.e., for all $x=\left(x_{n}\right) \in C(r, s), x_{i}=a_{i}$ for $r \leq i \leq s$. Let $\xi(i, j)$ be the collection of cylinders $C(i, j)$ and let $\xi_{j}=\xi(-j, j)$ for $j \in \mathbb{Z}^{+}$. A partition $\alpha$ of $\Omega$ is called a generator if $\bigvee_{i=0}^{\infty} F^{-i} \alpha \stackrel{B}{ }$ whenever

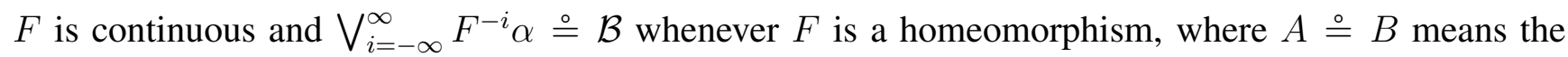
$\sigma$-algebra generated by $A$ coincides with the one generated by $B$ up to a measure zero set.

Lemma 1. Consider $(\Omega, F)$ a CA with local rule $f\left(x_{-1}, x_{0}, x_{1}\right)=\lambda_{-1} x_{-1}+\lambda_{0} x_{0}+\lambda_{1} x_{1} \bmod 2 . \xi_{j}$ is a generator for $j$ large enough provided $f$ is either one of the following cases:

(i) $F$ is either rule 170 or rule 240 , i.e., only $\lambda_{1} \neq 0$ or $\lambda_{-1} \neq 0$;

(ii) $F$ is either rule 90 or rule 150 , i.e., $\lambda_{-1}=\lambda_{1} \neq 0$.

Proof. First considering $F$ is rule 170, the case that $F$ is rule 240 can be demonstrated in analogous method.

$F$ is rule 170 indicates that $\lambda_{-1}=\lambda_{0}=0$, i.e., $F$ is the shift map. It comes immediately that $F^{-n} C(r, s)=C(r+n, s+n)$ for any cylinder $C(r, s)$ and $n \in \mathbb{N}$. Therefore,

$$
\bigvee_{i=0}^{n-1} F^{-i} \xi_{0}=\xi(0, n-1) \equiv\left\{{ }_{0}\left[a_{0} \ldots a_{n-1}\right]_{n-1}: a_{k}=0,1\right\}
$$

for $n \in \mathbb{Z}$, and

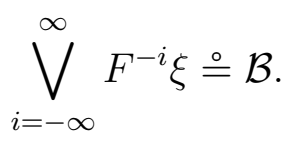

Thus $\xi_{0}$ is a generator.

If $F$ is rule 90 , then $\lambda_{-1}=\lambda_{1}=1$ and $\lambda_{0}=0$. Observe that

$$
\begin{aligned}
& F^{-1}[000]_{1}={ }_{-2}[00000]_{2} \cup_{-2}[01010]_{2} \cup_{-2}[10101]_{2} \cup{ }_{-2}[11111]_{2}, \\
& F^{-1}{ }_{-1}[001]_{1}={ }_{-2}[00001]_{2} \cup_{-2}[01011]_{2} \cup_{-2}[10100]_{2} \cup{ }_{-2}[11110]_{2}, \\
& F^{-1}{ }_{-1}[010]_{1}={ }_{-2}[00010]_{2} \cup{ }_{-2}[01000]_{2} \cup_{-2}[10111]_{2} \cup{ }_{-2}[11101]_{2}, \\
& F^{-1}{ }_{-1}[011]_{1}={ }_{-2}[00011]_{2} \cup{ }_{-2}[01001]_{2} \cup_{-2}[10110]_{2} \cup{ }_{-2}[11100]_{2}, \\
& F^{-1}{ }_{-1}[100]_{1}={ }_{-2}[00101]_{2} \cup_{-2}[01111]_{2} \cup{ }_{-2}[10000]_{2} \cup{ }_{-2}[11010]_{2}, \\
& F^{-1}{ }_{-1}[101]_{1}={ }_{-2}[00100]_{2} \cup_{-2}[01110]_{2} \cup_{-2}[10001]_{2} \cup{ }_{-2}[11011]_{2}, \\
& F^{-1}{ }_{-1}[110]_{1}={ }_{-2}[00111]_{2} \cup_{-2}[01101]_{2} \cup_{-2}[10010]_{2} \cup{ }_{-2}[11000]_{2}, \\
& F^{-1}{ }_{-1}[111]_{1}={ }_{-2}[00110]_{2} \cup_{-2}[01100]_{2} \cup_{-2}[10011]_{2} \cup \cup_{-2}[11001]_{2} .
\end{aligned}
$$

Thus $\xi_{1} \bigvee F^{-1} \xi_{1}=\xi_{2}$. By mathematical induction, $\bigvee_{i=0}^{n-1} F^{-i} \xi_{1}=\xi_{n}$ for $n \in \mathbb{N}$. Hence $\xi_{1}$ is a generator.

The case that $\lambda_{i}=1$ for $|i| \leq 1$ asserts that $\xi_{1}$ is a generator can be done via similar argument.

The proof is complete.

The measure-theoretic entropy of $F$ can be stated then. 
Theorem 2. Under the assumptions of Lemma 1, the measure-theoretic entropy of F can be expressed as the following.

$$
h_{\mu}(F)= \begin{cases}-2\left(p_{0} \log p_{0}+p_{1} \log p_{1}\right), & \lambda_{-1}=\lambda_{1}=1 \\ -\left(p_{0} \log p_{0}+p_{1} \log p_{1}\right), & \text { otherwise. }\end{cases}
$$

Proof. First considering either $\lambda_{-1}=0$ or $\lambda_{1}=0$, Lemma 1 and Kolmogorov-Sinai Theorem imply that

$$
h_{\mu}(F)=h_{\mu}\left(F, \xi_{0}\right)=\lim _{n \rightarrow \infty} \frac{1}{n} H\left(\bigvee_{i=0}^{n-1} F^{-i} \xi_{0}\right) \text {, }
$$

and

$$
\begin{aligned}
H\left(\bigvee_{i=0}^{n-1} F^{-i} \xi_{0}\right) & =H(\xi(0, n-1)) \\
& =-\sum_{i_{0}, \ldots, i_{n-1}} p_{i_{0}} p_{i_{1}} \cdots p_{i_{n-1}} \log \left(p_{i_{0}} p_{i_{1}} \cdots p_{i_{n-1}}\right) \\
& =-\sum_{i_{0}, \ldots, i_{n-2}} p_{i_{0}} \cdots p_{i_{n-2}} \sum_{i_{n-1}} p_{i_{n-1}}\left(\log p_{i_{0}}+\cdots+\log p_{i_{n-1}}\right) \\
& =-\left(\sum_{i_{0}, \ldots, i_{n-2}} p_{i_{0}} \cdots p_{i_{n-2}} \log p_{i_{0}} \cdots p_{i_{n-2}}+p_{0} \log p_{0}+p_{1} \log p_{1}\right) \\
& =-n\left(p_{0} \log p_{0}+p_{1} \log p_{1}\right) .
\end{aligned}
$$

This demonstrates that $h_{\mu}(F)=-\left(p_{0} \log p_{0}+p_{1} \log p_{1}\right)$.

Similarly, if $\lambda_{-1}=\lambda_{1}=1$, the proof of Lemma 1 asserts that $\xi_{1}$ is a generator and $\bigvee_{i=0}^{n-1} F^{-i} \xi_{1}=$ $\xi(-n, n)$. Hence

$$
\begin{aligned}
h_{\mu}(F) & =\lim _{n \rightarrow \infty} \frac{1}{n} H\left(\bigvee_{i=0}^{n-1} F^{-i} \xi_{1}\right) \\
& =-\lim _{n \rightarrow \infty} \frac{1}{n}(2 n+1)\left(p_{0} \log p_{0}+p_{1} \log p_{1}\right) \\
& =-2\left(p_{0} \log p_{0}+p_{1} \log p_{1}\right) .
\end{aligned}
$$

This completes the proof.

It is obvious that $h_{\mu}(F)=0$ if $\lambda_{-1}=\lambda_{1}=0$. For the case that $\lambda_{-1}=0$ and $\lambda_{0}=\lambda_{1}=1$, analogous argument as the proof of Lemma 1 shows that

$$
\bigvee_{i=0}^{n-1} F^{-i} \xi_{j}=\xi(-j, j+n-1), \quad \text { for } j \geq 1 .
$$

The following theorem comes immediately.

Theorem 3. If $F$ is either rule 60 or rule 102 , then $h_{\mu}(F)=-\left(p_{0} \log p_{0}+p_{1} \log p_{1}\right)$.

Proof. Without loss of generality, assume that $F$ is rule 60 , i.e., $\lambda_{-1}=0$. The other case can be done similarly.

Since $h_{\mu}(F)=\lim _{j \rightarrow \infty} h_{\mu}\left(F, \xi_{j}\right)$, above discussion establishes the desired formula. 
The measure-theoretic entropy of additive CA with nearest neighborhood can be concluded via Theorems 2 and 3.

Corollary 1. Let $(\Omega, F)$ be a CA with local rule $f\left(x_{-1}, x_{0}, x_{1}\right)=\lambda_{-1} x_{-1}+\lambda_{0} x_{0}+\lambda_{1} x_{1} \bmod 2$ and let $\mathcal{C}=\left\{i: \lambda_{i} \neq 0\right\} \bigcup\{0\}$. Then

$$
h_{\mu}(F)=-(M-m)\left(p_{0} \log p_{0}+p_{1} \log p_{1}\right)
$$

where $M=\max \mathcal{C}$ and $m=\min \mathcal{C}$.

\subsection{Topological entropy}

Let $\mathcal{O}$ be an open cover of $\Omega, \mathcal{O}$ is called a strong generator provided, for any $\delta>0$, there exists $N \in \mathbb{N}$ such that $\left\|\bigvee_{i=0}^{n-1} F^{-i} \mathcal{O}\right\|<\delta$ (respectively $\left\|\bigvee_{i=-n+1}^{n-1} F^{-i} \mathcal{O}\right\|<\delta$ ) for all $n \geq N$ whenever $F$ is continuous (respectively $F$ is homeomorphic), where $\|A\|$ is the diameter of $A$. In other words, $\mathcal{O}$ is a strong generator if and only if $\left\|\bigvee_{i=0}^{n-1} F^{-i} \mathcal{O}\right\| \rightarrow 0$ (or $\left\|\bigvee_{i=-n+1}^{n-1} F^{-i} \mathcal{O}\right\| \rightarrow 0$ ), as $n \rightarrow \infty$.

Since a cylinder is both open and close, $\xi_{j}$ is a finite open cover of $\Omega$ for $j \in \mathbb{N}$. The following lemma can be done via a slight change of the proof of Lemma 1 .

Lemma 2. Under the assumption of Lemma $1, \xi_{j}$ is a strong generator provided $j$ large enough.

A strong generator can be used for the calculation of topological entropy.

Theorem 4 ([28]). If $\xi$ is a strong generator of an endomorphism $(\Omega, F)$, then $h_{\text {top }}(F)=h_{\text {top }}(F, \xi)$.

Theorem 5. Under above assumption, let $M, m$ be the same as in Corollary 1 . Then $h_{\text {top }}(F)=(M-$ m) $\log 2$.

Proof. If $F$ is rule 170 , then $\xi_{0}$ is a strong generator since $\bigvee_{i=0}^{n-1} F^{-i} \xi_{0}=\xi(0, n)$ and $\bigvee_{i=-n+1}^{n-1} F^{-i} \xi_{0}=$ $\xi_{n}$. Theorem 4 implies that

$$
h_{\text {top }}(F)=\lim _{n \rightarrow \infty} \frac{1}{n} \log 2^{n+1}=\log 2 .
$$

Similarly, if $F$ is rule 90 , then $\xi_{1}$ is a strong generator and $\bigvee_{i=0}^{n-1} F^{-i} \xi_{1}=\xi_{n}$. It comes immediately that $h_{\text {top }}(F)=2 \log 2$.

The other cases can be done analogously. This completes the proof.

Moreover, under the same consideration of Theorem 3, the following theorem can be derived via a slight change of the proof.

Theorem 6. $h_{t o p}(F)=\log 2$.

The following corollary is a conclusion of Theorems 5 and 6, which can also be found in [24].

Corollary 2. If $F$ is additive, then $h_{t o p}(F)=(M-m) \log 2$.

Remark 1. Corollaries 2 and 2 demonstrate that the uniform Bernoulli measure is a measure with maximum entropy. 


\section{Ergodicity and Topological Pressure}

This section investigates the ergodicity of $F$ and the topological pressure.

\subsection{Ergodicity}

Shirvani and Rogers [19] demonstrate that a one-dimensional two states CA is ergodic provided its local rule is either rightmost or leftmost permutive. This fact has been rediscovered by Shereshevsky [20, 21] and Kleveland [22] for permutive CA. Cattaneo et al. [23] extend their result to multi-dimensional additive CA with arbitrary finite alphabet. A different proof for the ergodicity is given via a construction method.

Lemma 3 ([27]). Let $(X, \mathcal{B}, \mu)$ be a probability space and let $T: X \rightarrow X$ be a measure preserving transformation, then $T$ is ergodic if for $A, B \in \mathcal{B}, \mu(A), \mu(B)>0$, there exists $n \in \mathbb{N}$ such that $\mu\left(T^{-n} A \cap B\right)>0$.

Theorem 7. $F$ is ergodic except for $\lambda_{-1}=\lambda_{1}=0$.

Proof. It is obvious that $\mathrm{F}$ can not be ergodic if $\lambda_{-1}=\lambda_{1}=0$.

A scheme is constructed to show that $F$ is ergodic if $F$ is neither an identity map nor constant zero. Notably, it suffices to show that, for any two cylinder $C(p, q)={ }_{p}\left[c_{p}, \ldots, c_{q}\right]_{q}, D(r, s)={ }_{r}\left[d_{r}, \ldots, d_{s}\right]_{s}$, there exists $n \in \mathbb{N}$ such that $\mu\left(F^{-n} C(p, q) \cap D(r, s)\right)>0$, where $p \leq q, r \leq s$.

To make the scheme much easier to understand, the local rule $f\left(x_{-1}, x_{0}, x_{1}\right)=x_{0}+x_{1}$, i.e., rule 102 , is elucidated as an example.

The proof of Lemma 1 shows that $F^{-n} C(p, q) \in \xi(p, q+n)$ for $n \in \mathbb{N}$. It comes immediately that, if $s<p$ or $q<r$, then $\mu\left(F^{-1} C(p, q) \cap D(r, s)\right)>0$ since $F$ is rightmost permutive.

If $r \leq p \leq s \leq q$, define $\alpha_{11}, \alpha_{21}, \alpha_{22}, \ldots$ by

$$
\begin{aligned}
\alpha_{11} & =f_{1} \circ f_{2} \circ \cdots \circ f_{s-p}\left(d_{p}, d_{p+1}, \ldots, d_{s}\right), \\
\left(\alpha_{21}, \alpha_{22}\right) & =f_{2} \circ f_{3} \circ \cdots \circ f_{s-p}\left(d_{p}, d_{p+1}, \ldots, d_{s}\right), \\
\vdots & \\
\left(\alpha_{s-p, 1}, \ldots, \alpha_{s-p, s-p}\right) & =f_{s-p}\left(d_{p}, d_{p+1}, \ldots, d_{s}\right) .
\end{aligned}
$$

It is easily seen that there exists a unique $\beta_{11} \in \mathcal{A}$ such that $f\left(\alpha_{11}, \beta_{11}\right)=c_{p}$. Repeating the same process, there exist unique $\beta_{11}, \ldots, \beta_{1, q-p+1} \in \mathcal{A}$ such that $f_{q-p+1}\left(\alpha_{11}, \beta_{11}, \ldots, \beta_{1, q-p+1}\right)=\left(c_{p}, \ldots, c_{q}\right)$. Let $E_{1}={ }_{p}\left[\alpha_{11}, \beta_{11}, \ldots, \beta_{1, q-p+1}\right]_{q+1}$, then $F\left(E_{1}\right)=C(p, q)$.

Similarly, there exist unique $\beta_{21}, \ldots, \beta_{2, q-p+1} \in \mathcal{A}$ such that

$$
f_{q-p+2}\left(\alpha_{21}, \alpha_{22}, \beta_{21}, \ldots, \beta_{2, q-p+1}\right)=\left(\alpha_{11}, \beta_{11}, \ldots, \beta_{1, q-p+1}\right)
$$

and

$$
F\left(E_{2}\right)=E_{1}, \quad \text { where } E_{2}={ }_{p}\left[\alpha_{21}, \alpha_{22}, \beta_{21}, \ldots, \beta_{2, q-p+1}\right]_{q+2} \text {. }
$$

Construct $E_{1}, E_{2}, \ldots, E_{s-p}$ inductively so that $F^{i}\left(E_{i}\right)=C(p, q)$, for $1 \leq i \leq s-p$. Moreover, there exist unique $\beta_{s-p+1,1}, \ldots, \beta_{s-p+1, q-p+1} \in \mathcal{A}$ such that

$$
\begin{array}{r}
f_{q+s-2 p+1}\left(d_{p}, \ldots, d_{s}, \beta_{s-p+1,1}, \ldots, \beta_{s-p+1, q-p+1}\right) \\
=\left(\alpha_{s-p, 1}, \ldots, \alpha_{s-p, s-p}, \beta_{s-p, 1}, \ldots, \beta_{s-p, q-p+1}\right)
\end{array}
$$


and $F^{s-p+1}\left(E_{s-p+1}\right)=C(p, q)$, where

$$
E_{s-p+1}={ }_{p}\left[d_{p}, \ldots, d_{s}, \beta_{s-p+1,1}, \ldots, \beta_{s-p+1, q-p+1}\right]_{q+s-p+1} .
$$

Let $n=s-p+1$, then $\mu\left(F^{-n} C(p, q) \cap D(r, s)\right)>0$.

The other cases can be done analogously, thus is omitted. This completes the proof.

\subsection{Topological pressure}

For a given potential function $\phi \in C(\Omega, \mathbb{R})$, the topological entropy can be generalized to the consideration of topological pressure $P(F, \phi)$. It is obvious that $P(F, \phi)=0$ for all $\phi$ whenever $F$ is either zero or identity map. Let $a_{0}, a_{1} \in \mathbb{R}$ be given and let $\phi: \Omega \rightarrow \mathbb{R}$ be a potential function that depends on finitely many coordinates, the topological pressure $P(F, \phi)$ can be precisely formulated.

Potential functions depend on one coordinate

Let $\phi: \Omega \rightarrow \mathbb{R}$ be defined by $\phi(x)=a_{x_{0}}$, i.e., the potential of each $x \in \Omega$ is determined by its center coordinate.

Theorem 8. Let $M, m$ be the same as in Corollary 1 . If either $M$ or $m$ is nonzero, then $P(F, \phi)=$ $(M-m-1) \log 2+\log \left(e^{a_{0}}+e^{a_{1}}\right)$.

Proof. First considering that $\lambda_{-1}=0$ and $\lambda_{0}=\lambda_{1}=1$, since $\bigvee_{i=0}^{n-1} F^{-i} \xi_{j}=\xi(-j, n+j-1)$ for $n, j \in \mathbb{N}$,

$$
\begin{aligned}
p_{2}\left(F, \phi, \xi_{j}\right) & =\sum_{m_{1}, m_{2}} \sum_{A_{m_{1} ; m_{2}} \neq \varnothing} \sup _{x \in A_{m_{1} ; m_{2}}} \exp \left(\left(S_{2} \phi\right)(x)\right) \\
& =\sum_{m_{1}, m_{2}} \sum_{A_{m_{1} ; m_{2}} \neq \varnothing} \exp (\phi(x)+\phi(F(x))) \\
& =\sum_{i_{1}, i_{2} \in \mathcal{A}} 2^{2 j} \exp \left(a_{i_{1}}+a_{i_{2}}\right)=2^{2 j}\left(e^{a_{0}}+e^{a_{1}}\right)^{2},
\end{aligned}
$$

where $A_{m_{1} ; m_{2}} \in\left[m_{1}\right] \cap F^{-1}\left[m_{2}\right]$ and $m_{1}, m_{2} \in \mathcal{A}^{2 j+1}$. Mathematical induction asserts that

$$
p_{n}\left(F, \phi, \xi_{j}\right)=\sum_{i_{1}, \ldots, i_{n} \in \mathcal{A}} 2^{2 j} \exp \left(a_{i_{1}}+\cdots+a_{i_{n}}\right)=2^{2 j}\left(e^{a_{0}}+e^{a_{1}}\right)^{n}
$$

thus $P\left(F, \phi, \xi_{j}\right)=\log \left(e^{a_{0}}+e^{a_{1}}\right)$ for $j \in \mathbb{N}$. This demonstrates that $P(F, \phi)=\log \left(e^{a_{0}}+e^{a_{1}}\right)$.

The proof of other cases are similar, and the theorem follows.

The variational principle for topological pressure says that

$$
P(F, \phi)=\sup \left\{h_{\mu}(F)+\int_{\Omega} \phi d \mu: \mu \text { is an ergodic measure }\right\} .
$$

A measure $\mu$ is called an equilibrium measure provided $P(F, \phi)=h_{\mu}(F)+\int_{\Omega} \phi d \mu$. Corollary 1 and Theorem 8 are used for the determination of equilibrium measures. 
Example 1. Let $f\left(x_{-1}, x_{0}, x_{1}\right)=x_{-1}$, then $P(F, \phi)=\log \left(e^{a_{0}}+e^{a_{1}}\right)$ and

$$
h_{\mu}(F)+\int_{\Omega} \phi d \mu=-\sum_{i=0}^{1} p_{i} \log p_{i}+\sum_{i=0}^{1} a_{i} p_{i}=\sum_{i=0}^{1} p_{i}\left(a_{i}-\log p_{i}\right) .
$$

To determine whether $\mu$ is an equilibrium measure, define $\Phi:[0, \infty) \rightarrow \mathbb{R}$ by

$$
\Phi(x)= \begin{cases}0, & x=0 \\ x \log x, & \text { otherwise. }\end{cases}
$$

Then $\Phi$ is convex and $\Phi \in C^{1}((0, \infty), \mathbb{R})$. Moreover,

$$
\Phi\left(\sum_{i=1}^{n} \alpha_{i} x_{i}\right) \leq \sum_{i=1}^{n} \alpha_{i} \Phi\left(x_{i}\right), \quad \text { for } \sum_{i=1}^{n} \alpha_{i}=1, \alpha_{i} \geq 0, x_{i} \in \mathbb{R} .
$$

Let $\alpha_{i}=e^{a_{i}} / \lambda$ and $x_{i}=\left(p_{i} \lambda\right) / e^{a_{i}}$ for $i=0,1$, where $\lambda=e^{a_{0}}+e^{a_{1}}$.

$$
\begin{aligned}
0 & =\Phi(1)=\Phi\left(\sum_{i=0}^{1} \alpha_{i} x_{i}\right) \\
& \leq \sum_{i=0}^{1} \frac{e^{a_{i}}}{\lambda} \cdot \frac{p_{i} \lambda}{e^{a_{i}}} \log \frac{p_{i} \lambda}{e^{a_{i}}}=\sum_{i=0}^{1} p_{i} \log \frac{p_{i} \lambda}{e^{a_{i}}} \\
& =\log \left(e^{a_{0}}+e^{a_{1}}\right)-\sum_{i=0}^{1} p_{i}\left(a_{i}-\log p_{i}\right) .
\end{aligned}
$$

The equality holds if and only if $\left(p_{i} \lambda\right) / e^{a_{i}}=1$ for $i=0,1$, i.e., $\mu$ is an equilibrium measure if and only if $p_{i}=e^{a_{i}} /\left(e^{a_{0}}+e^{a_{1}}\right)$ for $i=0,1$.

Example 2. Consider Wolfram's rule 150, i.e., $f\left(x_{-1}, x_{0}, x_{1}\right)=x_{-1}+x_{0}+x_{1} \bmod 2$. Let $\phi: \Omega \rightarrow \mathbb{R}$ be defined by $\phi(x)=\log p_{x_{0}}$, then $P(F, \phi)=\log 2$ and

$$
h_{\mu}(F)+\int_{\Omega} \phi d \mu=-2 \sum_{i=0}^{1} p_{i} \log p_{i}+\int_{\Omega} \log p_{x_{0}} d \mu=-\left(p_{0} \log p_{0}+p_{1} \log p_{1}\right) .
$$

It is easily verified that $\mu$ is an equilibrium measure provided $\mu$ is the uniform Bernoulli measure.

Potential functions depend on finitely many coordinates

Let $\phi: \Omega \rightarrow \mathbb{R}$ be defined by $\phi(x)=a_{x_{k_{1}}} \cdots a_{x_{k_{2}}}$ for some $k_{1}, k_{2} \in \mathbb{Z}, k_{1} \leq k_{2}$, and let $\xi=\xi\left(k_{1}, k_{2}\right)$ be a finite partition. Define the transition matrix with respect to $\phi, \mathbf{T}_{\phi, F}=\left(t_{i j}\right)_{\left(k_{2}-k_{1}+1\right) \times\left(k_{2}-k_{1}+1\right)}$, by

$$
t_{i j}=s_{i j} \exp \phi(x), \quad x \in C_{i},
$$

where $C_{i}=\left[c_{0}, \ldots, c_{k_{2}-k_{1}}\right] \in \xi, i=1+\sum_{k=0}^{k_{2}-k_{1}} c_{k} \cdot 2^{k_{2}-k_{1}-k}$ and $s_{i j}$ denotes the cardinality of connected components of $C_{i} \cap F^{-1} C_{j}$. The following lemma comes immediately.

Lemma 4. Let $s=\max \left\{0, M+k_{1}-m-k_{2}-1\right\}$, then

$$
s_{i j}= \begin{cases}2^{s}, & C_{i} \cap F^{-1} C_{j} \neq \varnothing ; \\ 0, & \text { otherwise }\end{cases}
$$


Theorem 9. $P(F, \phi)=s \log 2+\log \rho$, where $\rho$ is the spectral radius of $2^{-s} \mathbf{T}_{\phi, F}$

Proof. The case that $\lambda_{-1}=0$ and $\lambda_{0}=\lambda_{1}=1$ is studied. The other cases can be done via analogous method, thus are omitted.

To clarify the proof, considering $k_{1}=0$ and $k_{2}=1$. Then $\xi=\left\{\left\{_{0}[00]_{1,0}[01]_{1},{ }_{0}[10]_{1},{ }_{0}[11]_{1}\right\}=\right.$ $\left\{C_{1}, C_{2}, C_{3}, C_{4}\right\}$, and

$$
\begin{array}{ll}
F^{-1} C_{1}={ }_{0}[000]_{2} \cup{ }_{0}[111]_{2}, & F^{-1} C_{2}={ }_{0}[001]_{2} \cup_{0}[110]_{2}, \\
F^{-1} C_{3}={ }_{0}[011]_{2} \cup_{0}[100]_{2}, & F^{-1} C_{4}={ }_{0}[010]_{2} \cup_{0}[101]_{2} .
\end{array}
$$

It is easily seen that

$$
s_{i j}= \begin{cases}1, & i=1,4, j=1,2 \\ 1, & i=2,3, j=3,4 \\ 0, & \text { otherwise. }\end{cases}
$$

and

$$
\mathbf{T}_{\phi, F}=\left(\begin{array}{cccc}
\exp \left(a_{0}^{2}\right) & \exp \left(a_{0}^{2}\right) & 0 & 0 \\
0 & 0 & \exp \left(a_{0} a_{1}\right) & \exp \left(a_{0} a_{1}\right) \\
0 & 0 & \exp \left(a_{0} a_{1}\right) & \exp \left(a_{0} a_{1}\right) \\
\exp \left(a_{1}^{2}\right) & \exp \left(a_{1}^{2}\right) & 0 & 0
\end{array}\right)
$$

Observe that $p_{1}(F, \phi, \xi)=\sum_{i_{1}, i_{2} \in \mathcal{A}} \exp \left(a_{i_{1}} a_{i_{2}}\right)=\left|\mathbf{T}_{\phi, F}\right| / 2$, where $|A|=\sum a_{i j}$ for $A$ a square matrix. Moreover, $p_{2}(F, \phi, \xi)=\sum_{m_{1}, m_{2} \in \mathcal{A}^{2}} \sum_{A_{m_{1} ; m_{2}} \neq \varnothing} \exp (\phi(x)+\phi(F(x)))=\left|\mathbf{T}_{\phi, F}^{2}\right| / 2$, where $A_{m_{1} ; m_{2}} \in$ $\left[m_{1}\right] \cap F^{-1}\left[m_{2}\right]$. It comes from induction that $p_{n}(F, \phi, \xi)=\left|\mathbf{T}_{\phi, F}^{n}\right| / 2$ for $n \in \mathbb{N}$, Perron-Frobenius theorem demonstrates that $P(F, \phi, \xi)=\log \rho$, where

$$
\rho=\frac{1}{2}\left(e^{a_{0}^{2}}+e^{a_{0} a_{1}}+\sqrt{\left(e^{a_{0}^{2}}-e^{a_{0} a_{1}}\right)^{2}+4 e^{a_{1}^{2}+a_{0} a_{1}}}\right)
$$

is the spectral radius of $\mathbf{T}_{\phi, F}$.

Furthermore, fixing $j \in \mathbb{N}$, then $p_{1}\left(F, \phi, \xi_{j}\right)=2^{2(j-1)}\left|\mathbf{T}_{\phi, F}\right|$ and $p_{n}\left(F, \phi, \xi_{j}\right)=2^{2(j-1)}\left|\mathbf{T}_{\phi, F}^{n}\right|$ for $n \in \mathbb{N}$. This infers that $P\left(F, \phi, \xi_{j}\right)=\log \rho$ for all $j \in \mathbb{N}$. The proof is done by letting $j$ tend to infinity.

Remark 2. It worth emphasizing that $\mathbf{T}_{\phi, F_{1}}$ and $\mathbf{T}_{\phi, F_{2}}$ are similar for any $f_{1}, f_{2} \in\left\{f=\sum_{i=-1}^{1} \lambda_{i} x_{i}\right.$ : $\left.\lambda_{-1}+\lambda_{1}=1\right\}$. Moreover, if $P$ is a four by four invertible matrix such that $\mathbf{T}_{\phi, F_{1}}=P^{-1} \mathbf{T}_{\phi, F_{2}} P$, then $P$ is the product of permutation matrices.

Remark 3. Theorem 8 can also be done via the same method as above. Whenever a potential function which depends only on one coordinate is considered, the transition matrix is universal for each local rule. That is,

$$
\mathbf{T}=2^{s}\left(\begin{array}{ll}
e^{a_{0}} & e^{a_{0}} \\
e^{a_{1}} & e^{a_{1}}
\end{array}\right)
$$




\section{Multiple Symbols and Larger Neighborhood}

This section generalizes those results in previous sections to the case that $|\mathcal{A}|=p$ and $f\left(x_{\ell}, \ldots, x_{r}\right)=$ $\sum_{i=\ell}^{r} \lambda_{i} x_{i} \bmod p$, where $p$ is prime. Without loss of generality, assume that $\lambda_{\ell}$ and $\lambda_{r}$ are both nonzero.

\subsection{Measure-theoretic entropy}

Let $\mu=\left(s_{0}, \ldots, s_{p-1}\right)$ be an $F$-invariant Bernoulli measure and let $\mathcal{C}, M$ and $m$ be the same as in Corollary 1. Analogous consideration still goes for the measure-theoretic entropy of additive CA.

Theorem 10. $h_{\mu}(F)=-(M-m) \sum_{i=0}^{p-1} s_{i} \log s_{i}$.

\subsection{Topological entropy}

Similar as discussed in last section, the argument in the proof of Theorem 5 gives an alternative proof for the formula that is demonstrated by Ward [24].

Theorem 11. $h_{\text {top }}(F)=(M-m) \log p$.

\subsection{Ergodicity and topological pressure}

Theorem 7 can be generalized via the same algorithm.

Theorem 12. $F$ is ergodic provided either $\lambda_{r} \neq 0$ with $r>0$ or $\lambda_{\ell} \neq 0$ with $\ell<0$.

Let $a_{0}, \ldots, a_{p-1} \in \mathbb{R}$ be given and let $\phi: \Omega \rightarrow \mathbb{R}$ be defined by $\phi(x)=a_{x_{0}}$. The following theorem is a general version of Theorem 8 .

\section{Theorem 13.}

$$
P(F, \phi)= \begin{cases}0, & M=m=0 \\ (M-m-1) \log p+\log \left(e^{a_{0}}+e^{a_{1}}+\cdots+e^{a_{p-1}}\right), & \text { otherwise }\end{cases}
$$

Furthermore, considering potential function $\phi(x)=\prod_{n=k_{1}}^{k_{2}} a_{x_{n}}^{b_{n}}$, where $k_{1}, k_{2} \in \mathbb{Z}^{+}, b_{n} \in \mathbb{R}$. Define

$$
\mathbf{T}_{\phi, F}=\left(t_{i j}\right)_{\left(k_{2}-k_{1}+1\right) \times\left(k_{2}-k_{1}+1\right)}, \quad t_{i j} \neq 0 \text { if and only if } C_{i} \cap F^{-1} C_{j} \neq \varnothing,
$$

where $C_{i} \in \xi(m, n)$ and $t_{i j}=\phi(x)\left(x \in C_{i}\right)$ provided $t_{i j} \neq 0$. Theorem 9 can be extended.

Theorem 14. Let $s=\max \left\{0, M+k_{1}-m-k_{2}-1\right\}$, then $P(F, \phi)=s \log p+\log \rho_{\mathbf{T}_{\phi, F}}$, where $\rho_{\mathbf{T}_{\phi, F}}$ is the spectral radius of $\mathbf{T}_{\phi, F}$.

It is worth emphasizing that, if either $M$ or $-m$ is greater than or equal to $k_{2}-k_{1}+1$, the topological pressure can be presented by

Corollary 3. $P(F, \phi)=s \log p+\log \sum \exp \left(a_{i_{1}} a_{i_{2}} \cdots a_{i_{k_{2}-k_{1}+1}}\right)$. 


\section{Acknowledgements}

The authors are grateful to anonymous referees for their valuable comments and suggestions.

\section{References and Notes}

1. Ulam, S. Random process and transformations. Proc. Int. Congress of Math. 1952, 2, 264-275.

2. von Neumann, J. Theory of self-reproducing automata. Univ. of Illinois Press, Urbana, 1966.

3. Bub, G.; Shrier, A.; Glass, L. Spiral wave generation in heterogeneous excitable media. Phys. Rev. Lett. 2002, 88, 058101.

4. Chernyak, Y.B.; Feldman, A.B.; Cohen, R.J. Correspondence between discrete and continuous models of excitable media: Trigger waves. Phys. Rev. E 1997, 55, 3125-3233.

5. Feldman, A.B.; Chernyak, Y.B.; Cohen, R.J. Wave-front propagation in a discrete model of excitable media. Phys. Rev. E 1998, 57, 7025-7040.

6. Greenberg, J.M.; Hassard, B.D.; Hastings, S.P. Pattern formation and periodic structure in systems modeled by reaction-diffusion equations. Bull. Amer. Math. Soc. 1978, 84, 1296-1327.

7. Richardson, D. Tessellation with local transformations. J. Compuut. System Sci. 1972, 6, 373-388.

8. Smith, III, A.R. Simple computational universal spaces. J. Assoc. Comput. Mach. 1971, 18, 339353.

9. Vichniac, G.Y. Boolean derivatives on cellular automata. Phys. D 1990, 45, 63-74.

10. Weimar, J.R.; Tyson, J.J.; Watson, L.T. Third generation cellular automaton for modeling excitable media. Phys. D 1992, 55, 328-339.

11. Wolfram, S. Statistical mechanics of cellular automata. Rev. Modern Physics 1983, 55, 601-644.

12. Hedlund, G.A. Endomorphisms and automorphisms of full shift dynamical system. Math. Systems Theory 1969, 3, 320-375.

13. Wolfram, S. Computation theory of cellular automata. Comm. Math. Phys. 1984, 96, 15-57.

14. Wolfram, S. Twenty problems in the theory of cellular automata. Phys. Scripta 1985, 9, 170-172.

15. Wolfram, S. A new kind of science. Wolfram Media, Champaign Illinois, USA, 2002.

16. Chua, L.O.; Sbitnev, V.I.; Yoon, S. A nonlinear dynamics perspective of Wolfram's new kind of science. Part III: Predicting the unpredictable. Int. J. Bifurcation and Chaos 2004, 14, 3689-3820.

17. Chua, L.O.; Guan, J.; Sbitnev, V.I.; Jinwook, S. A nonlinear dynamics perspective of Wolfram's new kind of science. Part VII: Isles of eden. Int. J. Bifurcation and Chaos 2007, 17, 2839-3012.

18. Chua, L.O.; Karacs, K.; Sbitnev, V.I.; Guan, J.; Jinwook, S. A nonlinear dynamics perspective of Wolfram's new kind of science. Part VIII: More isles of eden. Int. J. Bifurcation and Chaos 2007, 17, 3741-3894.

19. Shirvani, M.; Rogers, T.D. On ergodic one-dimensional cellular automata. Commun. Math. Phys. 1991, 136, 599-605.

20. Shereshevsky, M.A. K-property of permutative cellular automata. Indag. Math. (N.S.) 1997, $8,411-416$.

21. Shereshevsky, M.A. Ergodic properties of certain surjective cellular automata. Monatsh. Math. 1992, 114, 305-316. 
22. Kleveland, R. Mixing properties of one-dimensional cellular automata. Proc. Amer. Math. Soc. 1997, 125, 1755-1766.

23. Cattaneo, G.; Formenti, E.; Manzini, G.; Margara, L. Ergodicity, transitivity, and regularity for linear cellular automata over $z_{m}$. Theoret. Comput. Sci. 2000, 233, 147-164.

24. Ward, T. Additive cellular automata and volume growth. Entropy 2000, 2, 142-167.

25. Akın, H. On the measure entropy of additive cellular automata $f_{\infty}$. Entropy 2003, 5, 233-238.

26. Berg, K.R. Convolution of invariant measures, maximal entropy. Math. Systems Theory 1969, 3, 146-150.

27. Walters, P. An introduction to ergodic theory. Springer-Verlag: New York, 1982.

28. Pollicott, M.; Yuri, M. Dynamical systems and ergodic theory. Cambridge University Press: 1998.

(c) 2009 by the authors; licensee Molecular Diversity Preservation International, Basel, Switzerland. This article is an open-access article distributed under the terms and conditions of the Creative Commons Attribution license (http://creativecommons.org/licenses/by/3.0/). 